\title{
Estudo da dispersão de monóxido de carbono emitido por queimadas na Amazônia legal em 19 agosto de 2010 baseado em: simulações do modelo WRF-CHEM e SensoriamentoRemoto.
}

\author{
Study of Dispersion of Carbon Monoxide emitted by biomass burning in the Amazon \\ on August 19, 2010 based on WRF-CHEM simulation And Remote Sensing.
}

\author{
Ricardo Antonio Mollmann Junior ${ }^{1}$, Rosiberto Salustiano da Silva Júnior ${ }^{1}$, \\ Simone Marilene Sievert da Costa Coelho ${ }^{2}$ e Bruno Lisbôa Medina ${ }^{2}$ \\ ${ }^{1}$ Instituto de Ciências Atmosféricas, Universidade Federal de Alagoas Maceió, Brasil \\ ${ }^{2}$ Divisão de Satélites e Sistemas Ambientais, Instituto Nacional de Ciências Atmosféricas, Cachoeira Paulista, \\ Brasil
}

\begin{abstract}
Resumo
A Região Amazônica, apresenta um ecossistema tropical único que ainda preserva grande parte de sua floresta nativa, no entanto, vem sofrendo extensivas mudanças devido a constante presença de queimadas nessa região. O módulo químico do modelo atmosférico WRF (WRF/CHEM - Weather Reaserch Forecasting/CHEMistry), possibilitou a análise da concentração e dispersão do gás traço Monóxido de Carbono (CO), oriundos dos focos de queimadas detectadas operacionalmente no Instituto Nacional de Pesquisas Espaciais(INPE), pelos sensores AVHRR dos satélites polares NOAA-15, NOAA-16, NOAA-17, NOAA-18 e NOAA-19; as imagens do sensor MODIS nos satélites polares da NASA: TERRA e AQUA; $e$ as imagens dos satélites geoestacionários GOES- 12 e MSG-2, no dia 19 de Agosto de 2010. Para avaliar a dispersão foi utilizada a direção e a velocidade do vento a 10 metros de altura, no sentido de estimar o destino da pluma de gás emitida pela queimada, é ainda feita uma análise do transporte do gás traço na coluna atmosférica através de sensoriamento remoto com informações do instrumento AIRS (Atmospheric Infrared Sounder) a bordo do satélite AQUA. Verificou-se que o CO pode afetar a qualidade do ar local ao assumir altas concentrações a poucos quilômetros da fonte de emissão (aproximadamente 2000 ppbv), porém com a dispersão do gás em função do vento, é possível ver concentraçães próximas à 500 ppbv a pelo menos $300 \mathrm{~km}$ da fonte de emissão, com o poluente sendo transportado para outros países da América do sul.
\end{abstract}

Palavras-chave: Queimadas, Região Amazônica, Modelagem Atmosférica, Poluição Atmosférica, Sensoriamento Remoto.

\begin{abstract}
The Amazon region has a unique tropical ecosystem that still preserves much of its native forest, however, has undergone extensive changes due to the constant presence of fire in the region. The chemical module of the atmospheric model WRF (WRF/CHEM - Weather Reaserch Forecasting/CHEMistry), allowed analysis of the concentration and dispersion of the trace gas Carbon Monoxide (CO), arising from burning source detected operationally in the National Institute for Space Research (INPE), by AVHRR sensors of the NOAA-15, NOAA16, NOAA-17, NOAA-18 and NOAA-19 polar satellites; images of MODIS sensor on NASA's polar satellites TERRA and AQUA, and the images of the geostationary satellites GOES-12 and MSG-2, on August 19, 2010. To evaluate the dispersion wind direction and was used at 10 meters high, in order to estimate the destination of the gas plume emitted by the forest fire, is also an analysis of the transport of atmospheric trace gas column through remote sensing with instrument information AIRS (Atmospheric Infrared Sounder) aboard the AQUA satellite. It was seen CO can affect local air quality by taking high concentrations within a few kilometers of the emission source (approximately $2000 \mathrm{ppbv})$, but with the dispersion of the gas due to the wind, it is possible to see concentrations close to $500 \mathrm{ppbv}$ at least $300 \mathrm{~km}$ from the emission source, with transport of pollutant reaching other countries of South America.
\end{abstract}




\section{Introdução}

A Região Amazônica possui um ecossistema tropical único que ainda preserva grande parte de sua floresta nativa, no entanto, vem sofrendo extensivas mudanças no uso do solo (Gash et al., 1996; Artaxo et al., 2001). A chamada Amazônia Legal é uma área que engloba nove estados brasileiros pertencentes à Bacia Amazônica, ocupa uma área de $5.016 .136,3 \mathrm{~km}^{2}$, que correspondem a cerca de $59 \%$ do território brasileiro.

O uso do fogo é um procedimento agrícola muito utilizado nessa região, por diversos sistemas de produção. No vasto domínio das culturas agrícolas existentes na Amazônia, o fogo pode estar associado: ao desmatamento, à renovação de pastagens, ao manejo de capoeiras, à eliminação de resíduos agrícolas, ao controle de pragas, à colheita da cana-de-açúcar e do algodão etc. (EMBRAPA, 2006).

De acordo com Freitas (2005), os estágios de evolução de uma queimada são descrito em quatro fases: ignição, chamas, brasas e extinção. Onde a ignição é dependente do tipo de biomassa, da umidade e de fatores ambientais, como temperatura, umidade relativa e o vento. O estágio de chamas é onde as altas temperaturas provocam uma ruptura das moléculas constituintes da biomassa, onde componentes com alto peso molecular são decompostos em compostos com peso molecular mais baixo tais como o carvão e o alcatrão, os quais constituem fonte primária de energia para as chamas, e finalmente em compostos de natureza gasosa. É nessa fase onde se inicia a liberação de compostos como o $\mathrm{CO}$ e o $\mathrm{CO}_{2}$. Com a diminuição das condições necessárias para se manter as chamas, a queima entra em um estágio mais "frio", chamado de fase de brasas, com isso reduz drasticamente a produção de $\mathrm{CO}_{2}$, e há um aumento na emissão de compostos oxidados, como o $\mathrm{CO}$, além de uma rápida formação de partículas por acreção de partículas orgânicas de carbono. Finalmente, a fase de extinção onde é alcançada devido principalmente à diminuição da quantidade de biomassa disponível.

Na Amazônia, movimentos convectivos frequentes influenciam significativamente o transporte de gases e partículas de aerossóis emitidos por queimadas (F REITAS, 1999 apud A RTAXO, 2004). O transporte a longa distância de gases traço e partículas de aerossol segue o padrão de circulação atmosférica, fazendo com que as emissões da floresta amazônica sejam observadas a centenas de quilômetros da floresta.

Atualmente, estudos referentes ao comportamento meteorológico e químico de fontes de poluição incluindo queima de biomassa e ao transporte de gases partículas de aerossol, vêm sendo tratados por meio de modelos computacionais acoplados, que simulam os impactos causados na atmosfera de determinadas emissões por diversos poluentes. Uma das grandes dificuldades dos modelos que estudam a dispersão dos poluentes seria a determinação dos focos de queimadas e a taxa de emissão do poluente para à atmosfera.

Em um estudo para determinar os impactos da queima de biomassa no Alaska, Greell et al. (2010), fez a inclusão de um modelo de emissão por queima de biomassa, $\mathrm{Bra}$ zilian Biomass Burning Emission (3BEM), que se baseia em produtos de sensoriamento remoto em tempo quase real para determinar as emissões de queimadas (FREITAS et al., 2005; LONGO et al., 2007). No 3BEM as emissões de fogo são atualizadas à medida que são disponíveis, e são distribuídas espacial e temporalmente de acordo com os locais de queimadas obtido por sensoriamento remoto (oriundos dos sensores AVHRR, MODIS e GOES12). Para este estudo, o método para obtenção dos focos de queimadas adotado foi de modo similar ao estudo de Grell et al.

Em 1991, Setzer e Pereira implementaram de forma operacional no Instituto Nacional de Pesquisas Espaciais (INPE), a técnica de detecção de focos de queimadas na região do cerrado e floresta tropical no Brasil usando produtos do radiômetro AVHRR (Advanced Very High Resolution Radiometer) a bordo dos satélites polares NOAA-15, NOAA-16, NOAA-17, NOAA-18 e NOAA-19. Atualmente, o produto de queimadas engloba sensores a bordo de satélites ambientais e meteorológicos, como: o radiômetro AVHRR da série de satélites da NOAA; o sensor MODIS (Moderate Resolution Imaging Spectroradiometer) dos satélites polares da NASA: TERRA e AQUA; e os satélites geoestacionários GOES- 2 e MSG-2. Esses produtos são disponibilizados em tempo quase real no site do INPE (http://www.dpi.inpe.br/proarco/ bdqueimadas/).

Além da detecção e caracterização dos focos de incêndio, têm-se desenvolvido técnicas de sensoriamento remoto destinadas a observar e quantificar os efeitos dos produtos emitidos. O satélite AQUA realiza medições de gases traços na atmosfera. A medição das concentrações de monóxido de carbono na atmosfera através de um instrumento chamado AIRS (Atmospheric Infrared Sounder), que é baseado na radiação a comprimentos de onda específicos associados a absorção por concentrações de monóxido de carbono. O AIRS é sensível ao CO na troposfera média a alturas entre 2 e 10 quilômetros, com um pico de sensibilidade em uma altitude de aproximadamente 5 quilômetros. Essa região da atmosfera da Terra também é propícia para o transporte de longa distância da poluição que é levada para esta altitude.

Este estudo tem o objetivo de observar a dispersão do gás traço Monóxido de Carbono emitido por queimada na região da Amazônia Legal associado à direção e a velocidade do vento a 10 metros de altura, no sentido de estimar o destino da pluma de gás emitida pela queimada, durante a temporada de queimadas na região, em um dia do mês de Agosto de 2010. E ainda a confirmação dos resultados obtidos com dados de sensoriamento remoto. 


\section{Materiais e Métodos}

\section{1 Área de Estudo}

A área que corresponde a chamada Amazônia Legal corresponde à totalidade dos estado do Acre, Amapá, Amazonas, Pará, Rondônia, Roraima e Tocantins, e parte dos estado do Mato Grosso e Maranhão, perfazendo uma superfície de aproximadamente $5.271 .423 \mathrm{~km}^{2}$, correspondendo a 59\% do território brasileiro (Figura 1). Entretanto, sua população corresponde a $12,32 \%$ do total de habitantes do Brasil (IBGE, 2010).

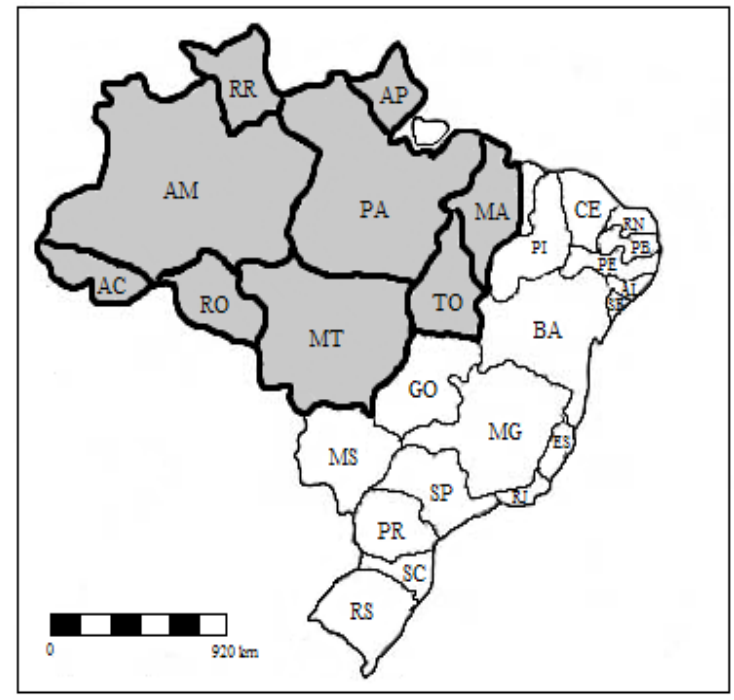

Figura 1 - Área em destaque, correspondente a Amazônia Legal no território brasileiro

Fonte: Autor, 2013
A sazonalidade climática da Amazônia bem marcada, a estação seca se intensifica no norte da região, durante os meses de janeiro, fevereiro e março, enquanto a porção sul sofre com períodos secos durante os meses de julho, agosto e setembro. As queimadas seguem claramente o mesmo padrão das chuvas (ARAGÃO et al. 2009).

Apesar de serem detectadas durante todo o ano, são mais frequentes no primeiro trimestre na região norte e no terceiro na região sul e leste.

A escolha de um dia durante o ano de 2010, foi devido ao aumento significativo na quantidade de focos no ano, principalmente durantes o meses de Agosto e Setembro, em comparação aos anos anteriores (Figura 2). Sendo que o mês de Agosto é o início da estação seca e consequentemente do período de queimadas na Região da Amazônia Legal. Apenas no dia de estudo, 19 de Agosto de 2010, foram registrados pelos sensores orbitais, 4.451 focos de queimadas na região.

\subsection{Configurações do Modelo WRF/CHEM}

O modelo WRF/Chem é um modelo meteorológico acoplado com um módulo químico, que possibilita fazer testes de dispersão de poluentes utilizando um traçador atmosférico. O modelo foi configurado com um domínio de 228 x 178 pontos em x e y, 27 níveis verticais e uma resolução espacial horizontal de $12 \mathrm{~km}$, suficiente para um estudo de dispersão de poluentes na região.

As simulações foram iniciadas para o intervalo de 24 horas, as 00:00Z do dia 19 de Agosto de 2010, com saídas do modelo para cada hora do dia. As condições iniciais e de contorno derivam do modelo GFS (Global Forecast System) executado pelos centros de previsão nacional

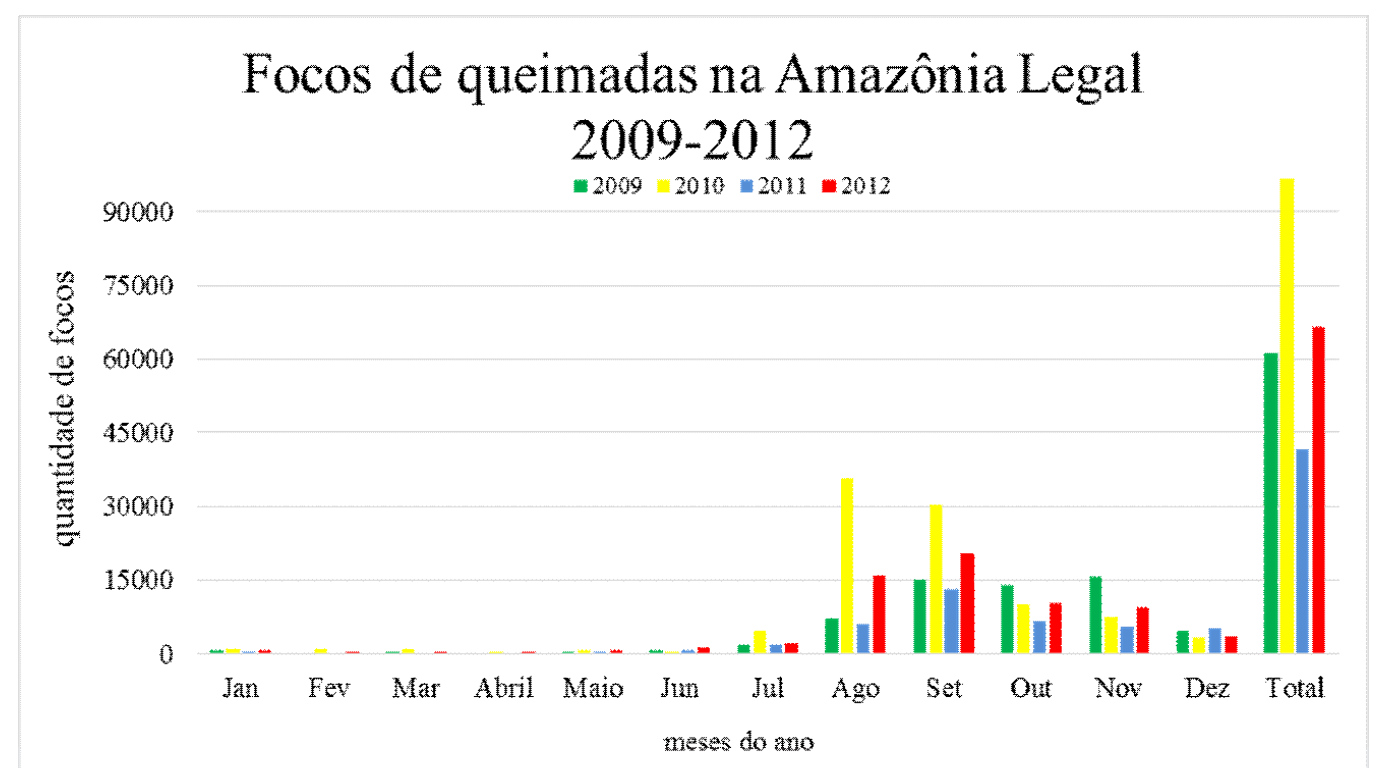

Figura 2 - Focos de queimadas na Região da Amazônia Legal durante o período de 2009 a 2012. A quantidade de focos é baseado na quantidade contabilizada pelo satélite de referência (NOAA-15 até agosto de 2011, a partir de Setembro de 2011, passou a ser o satélite AQUA-MODIS). FONTE: (http://www.dpi.inpe.br/proarco/bdqueimadas/). 
(NCEP), o qual é uma filiar da NOAA (National Oceanic and Atmospheric Administration), do NWS (National Weather Service), nos EUA.

O poluente químico usado como gás traçador foi o Monóxido de Carbono (CO). A escolha do CO para análise da simulação se deve ao fato do mesmo ser um poluente produzido pela combustão incompleta na queima de biomassa e de combustíveis fósseis, além de ser pouco reativo depois de emitido na atmosfera. O CO é um gás tóxico incolor e inodoro. Quando a fonte emissora é a queima de biomassa, o CO é produzido principalmente no início da combustão com ausência de chamas.

A quantidade emitida de Monóxido de Carbono em cada foco de queimada foi obtida através do método definido por Freitas et al. (2005) e Longo et al. (2007). Este método determina que para cada foco a massa do traçador emitida é calculada pela equação (1), que leva em consideração os valores estimados para a quantidade de biomassa acima do solo disponível para a queima $(\alpha)$, o fator de combustão $(\beta)$ e o fator de emissão $\left(E_{f}\right)$

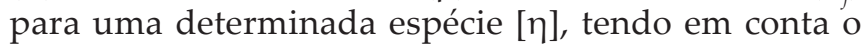
tipo de vegetação, e a área de gravação (a) para cada evento de queima.

$$
\mathrm{M}[\eta]=\alpha v e g \cdot \beta v e g . \operatorname{Ef}[\eta] \text {.afire }
$$

Os parâmetros $\left(\alpha_{v e g} \cdot \beta_{v e g} \cdot E_{f}^{[\eta]}\right)$ adotado neste estudo são aqueles propostos por Ândreae e Merlet (2001) para Floresta Tropical, sendo: fator de emissão $\left(E_{f}\right)$ para o $C O$ igual a $110 \mathrm{~g} / \mathrm{kg}$, a quantidade de biomassa acima do solo disponível para a queima $(\alpha)$ acima do solo igual a $20.7 \mathrm{~kg} / \mathrm{m}^{2}$ e o Fator de Combustão $(\beta)$ igual 0.48 , sendo a área de queimada $\left(\mathrm{a}_{\text {fire }}\right)$ a área da grade do modelo.

\subsection{Dados de Sensoriamento Remoto}

Conforme descrito no portal de queimadas do INPE, para a detecção dos focos de queimadas é feita a análise em que um material em chamas, ou seja, no processo de queima de biomassa, é emite energia eletromagnética de 3,7 $\mu \mathrm{m}$ a $4,1 \mu \mathrm{m}$, principalmente na faixa térmica. O CPTEC/INPE identifica através de sensoriamento remoto as emissões nessa faixa, assumindo como focos de queimadas.

E assim utilizando as imagens de diversos satélites (AVHRR, NOAA e MODIS), que operam neste espectro, é selecionando o pixel (elementos de resolução) com maior temperatura e obtidos assim os focos de queimadas. Depois de analisadas os dados da localização dos focos de queimadas e georreferenciados, os dados são inseridos no modelo WRF/Chem (Weather Research and Forecasting/Chemistry), (ver figura 3). Esses focos foram retirados do site do INPE referente ao dia 19 de Agosto 2010 na região da Amazônia Legal.

Com base em dados de sensoriamento remoto referentes a qualidade do ar da NASA, da divisão GIOVANNI (GES-DISC (Goddard Earth Sciences Data and Information Services Center) Interactive Online Visualization And aNalysis Infrastructure) do Goddard Earth Sciences (GES) Data and Information Services Center (DISC) (http://disc.sci. gsfc.nasa.gov/giovanni). Foi analisado dados do satélite AQUA referente a média zonal da concentração de $\mathrm{CO}$, integrado na coluna atmosférica, sobre área da Amazônia Legal do ano 2009 até 2012 (Figura 4).

É possível notar nesta figura que existe um ciclo sazonal bem definido nas concentrações de $\mathrm{CO}$, com máximos valores ocorrendo nos meses de agosto a ou-
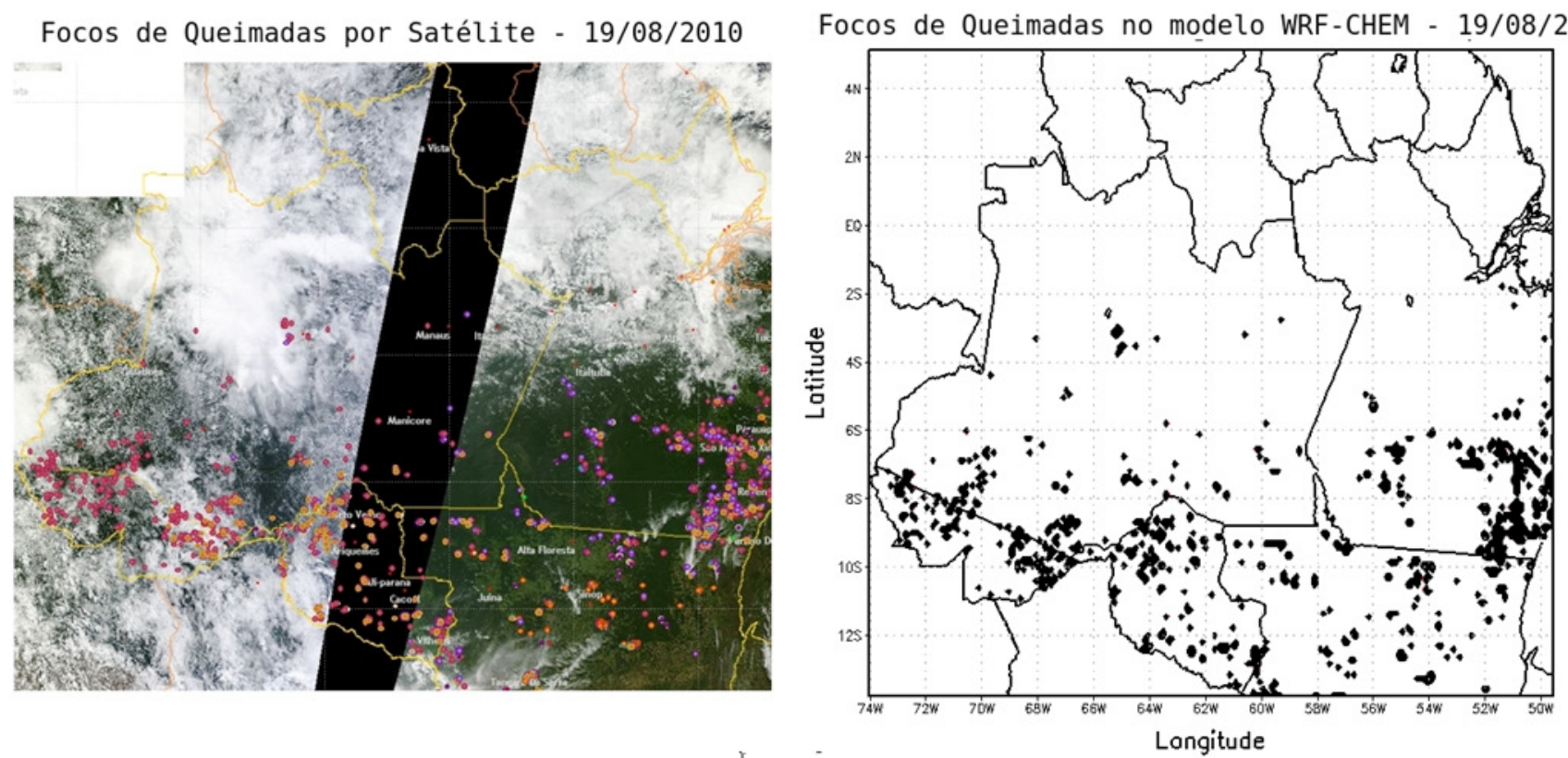

Figura 3 - Focos de queimadas para o 19 de agosto 2010 (Fonte: www.cptec.inpe.br/queimadas). (a) Os pontos de queimadas identificados pelo satélite são representados pelos pontos coloridos, os pontos em vermelho são as representações municipais, (b) Focos de queimadas inseridos no modelo WRF-CHEM 


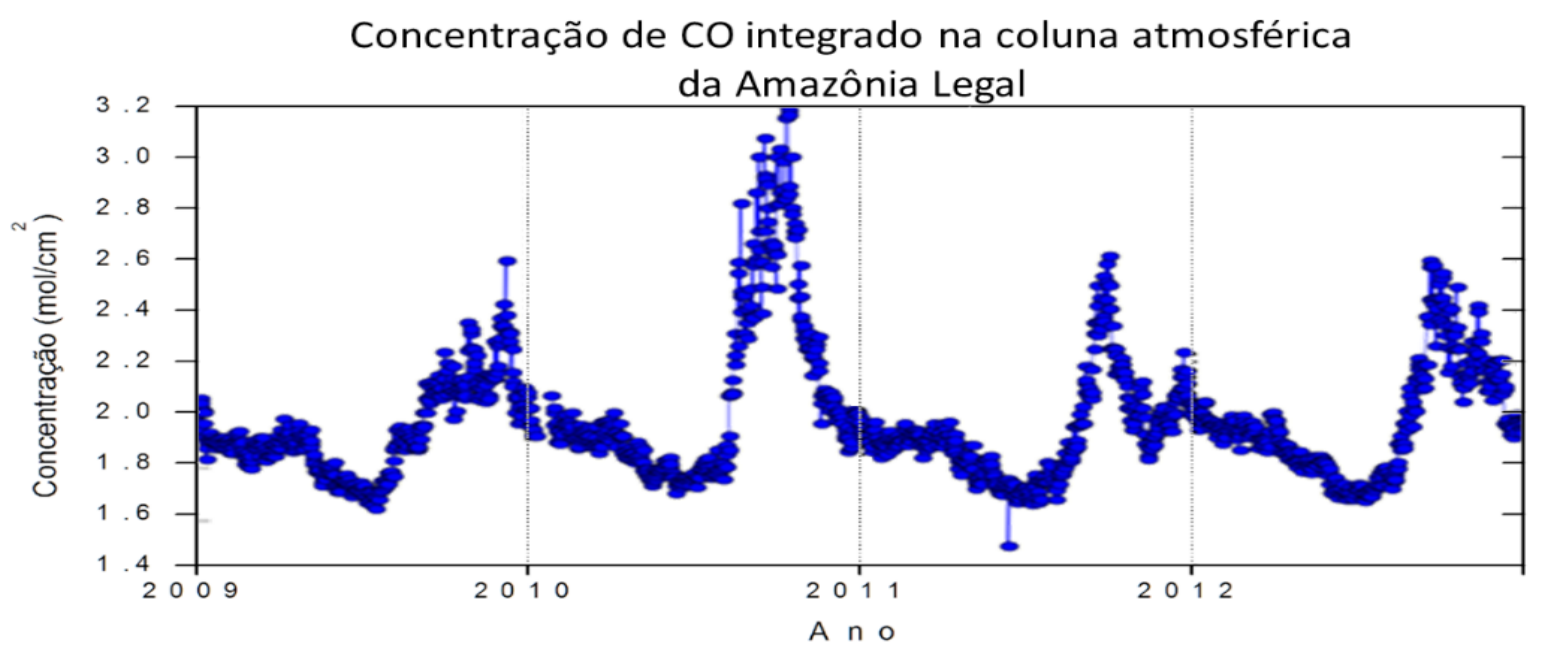

Figura 4 - Concentração de CO integrado na coluna atmosférica para a região da Amazônia Legal para o período de 2009 a 2012

tubro, que correspondem ao período da estação seca sobre a região estudada.

É visto ainda, que o ano de 2010 foi o período que se obteve os maiores valores de moléculas de CO na coluna atmosférica, sendo o seu período seco encontrado os maiores picos.

\section{Resultados e Discussões}

Nesta seção serão apresentados os resultados obtidos nas simulações com o modelo WRF/CHEM, com os ajustes em termos de posicionamento dos focos de queimadas e estimativas feitas quanto a emissão de $\mathrm{CO}$ por queima de biomassa, para o dia 19 de Agosto de 2010.

\subsection{Caracterizações da Dispersão dos Poluentes}

É possível acompanhar o início das emissões à 01:00Z do 19 de Agosto (Figura 5A), onde quase toda totalidade dos focos são observados na parte mais ao sul da região da Amazônia Legal, entre o sul estado do Amazonas, Acre, Rondônia, sudeste do Pará e norte do Mato Grosso. Nota-se uma atividade do vento baixa nessa região.

Porém, às 05:00Z (Figura 5B), observar-se um aumento na velocidade do vento $(6 \mathrm{~m} / \mathrm{s})$ principalmente a leste da região, ao sul do estado do Pará e norte do Mato Grosso, onde nota-se um sentido zonal dominante na direção do vento nessa região, de leste para oeste, isto faz com que o poluente emitido se desloque rapidamente de sua fonte. A oeste, na Figura 5B, na divisa entre os estados do Acre, Rondônia e Amazonas é possível observar uma confluência de ventos fracos. Isso acarreta numa estagnação do poluente sobre determinada região, podendo observar áreas com altos valores de concentração de CO (1400 ppbv).
Na imagem das 10:00Z (Figura 5C) é observado uma diminuição no padrão zonal do vento, assumindo um sentido de sudeste para noroeste, visto na parte sudeste da região. Também ocorre a diminuição na velocidade do vento sobre essa região, fazendo que a concentração próxima a fonte de emissão tenha maiores valores, e a dispersão se mantenha em pontos mais próximos aos focos. A oeste, é observado uma vasta área de confluência de ventos, sobre o estado de Rondônia e fronteira dos estados do Acre e Amazonas, isso ocasiona aprisionamento do CO emitido naquela região, caracterizando maiores concentrações do poluente, na ordem de 1800 ppbv.

Às 15:00Z (Figura 5D), é possível novamente observar ventos com velocidades suficiente para dispersarem o $\mathrm{CO}$ de seus devidos focos deixando concentrações na ordem de 800 a 500 ppbv. É possível notar este aumento das velocidades em todas as áreas de focos, principalmente no focos a oeste, entre a fronteira do Acre e o estado do Amazonas, nessa imagem começa a se notar uma dispersão do gás para fora do território do Brasil, chegando até o Peru.

Às 19:00Z (Figura 5E), é visto novamente uma diminuição na velocidade do vento, isso ocasiona novamente em estagnação do CO próximo aos focos, assumindo assim altos valores de concentração. Ainda, também é possível notar uma pequena concentração de Monóxido de Carbono no território peruano, entre 800 a 500 ppbv.

$\mathrm{Na}$ última hora da rodada (Figura $5 \mathrm{~F}$ ), notamos um padrão parecido ao anterior, com as concentrações dispersas por grandes áreas ao redor do território da Amazônia Legal, com concentrações chegando a 1800 ppbv em algumas áreas próximo as fontes, e a pelo menos a 500 ppbv a quilômetros de distância do foco de emissão, como é visto nas concentrações que atravessam o território do Peru e chegam até a Colômbia, percorrendo um distância de pelo menos $300 \mathrm{~km}$. 

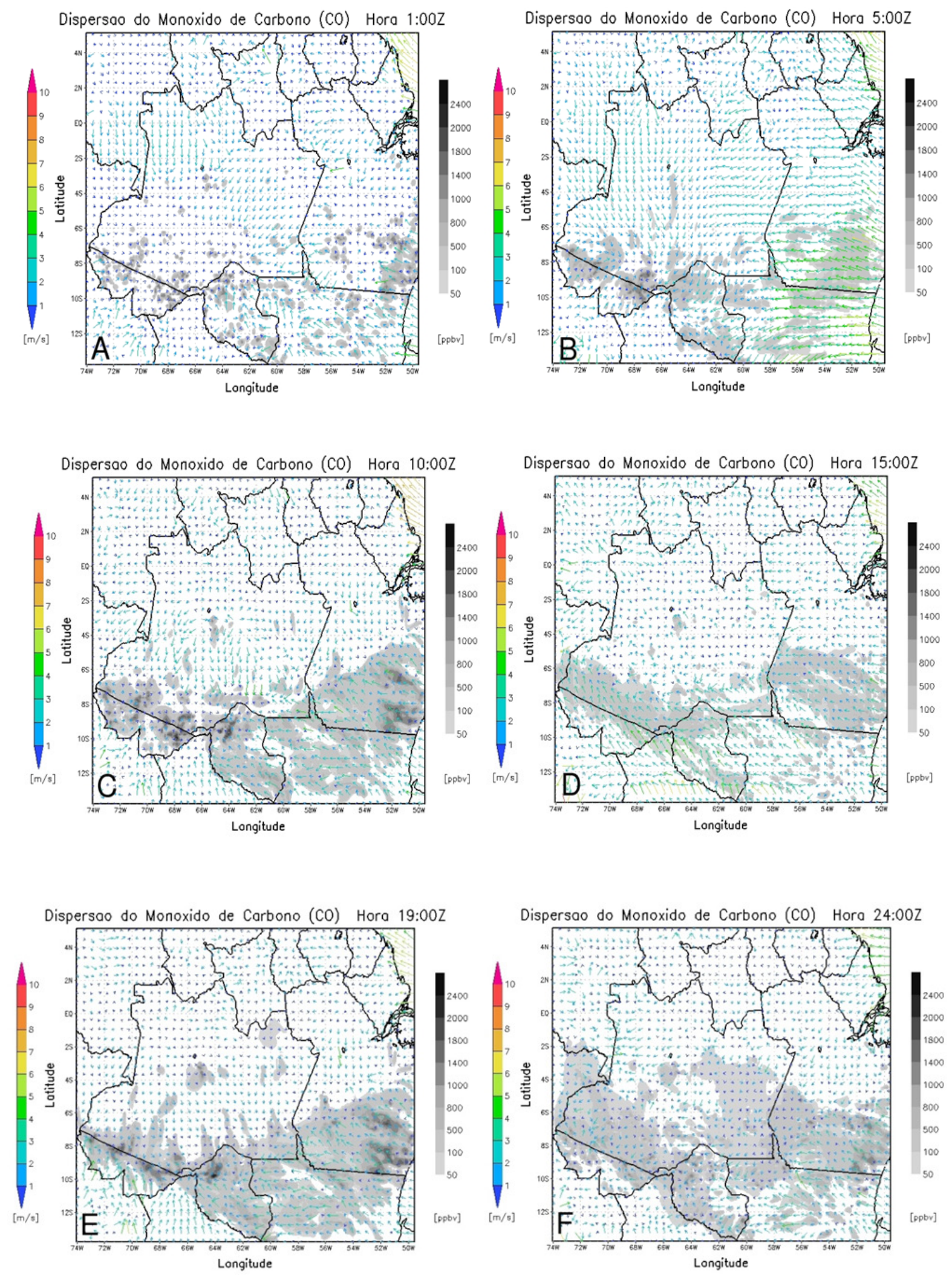

Figura 5 - Dispersão de Monóxido de Carbono associado ao vento em 10 metros no dia 19 de Agosto as (A) 01:00, (B) 05:00, (C) 10:00 e (D) 15:00, (E) 19:00, (F) 24:00 Z 


\subsection{Análise da Dispersão através de Sensoria- mento Remoto}

Para uma análise referente ao transporte do gás Monóxido Carbono foi comparado a média de dispersão do modelo WRF/CHEM sobre a região da Amazônia, à dados de sondagem do sensor Atmospheric Infrared Sounder (AIRS) presente no satélite AQUA, e faz parte da aplicação GIOVANNI desenvolvida pela GES DISC: (Goddard Earth Sciences, Data and Information Services Center) (Figura 6). É possível notar um bom desempenho do modelo para representar o transporte do poluente. Observa-se a dispersão se dando no sentido de sudeste para noroeste da região, migrando do território brasileiro para os países do Peru e Colômbia, as imagens mostram esse padrão.

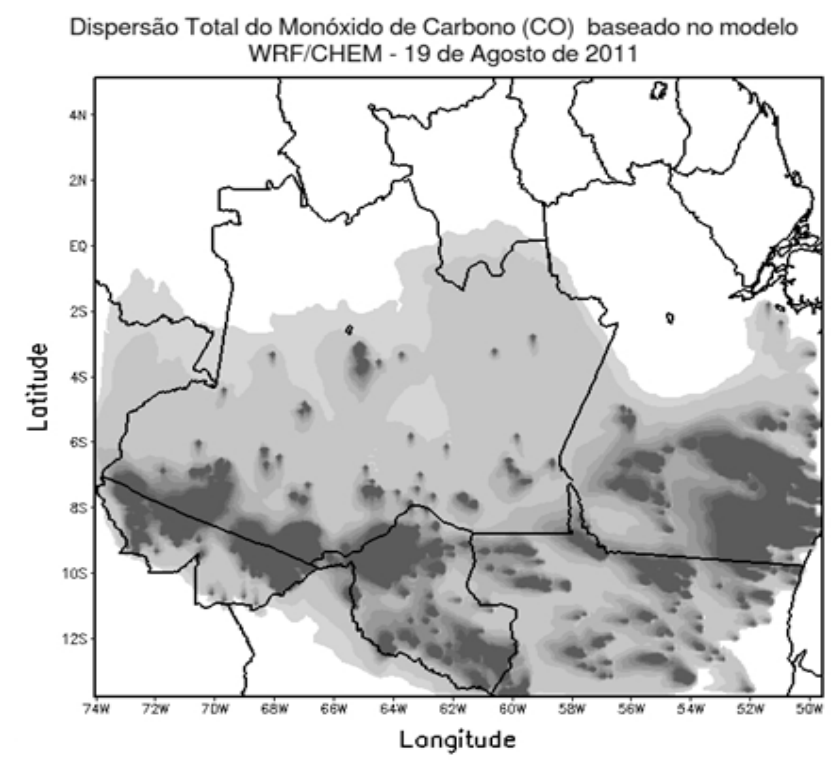

Total ascendente de Monóxido de Carbono (CO) na coluna atmosférica

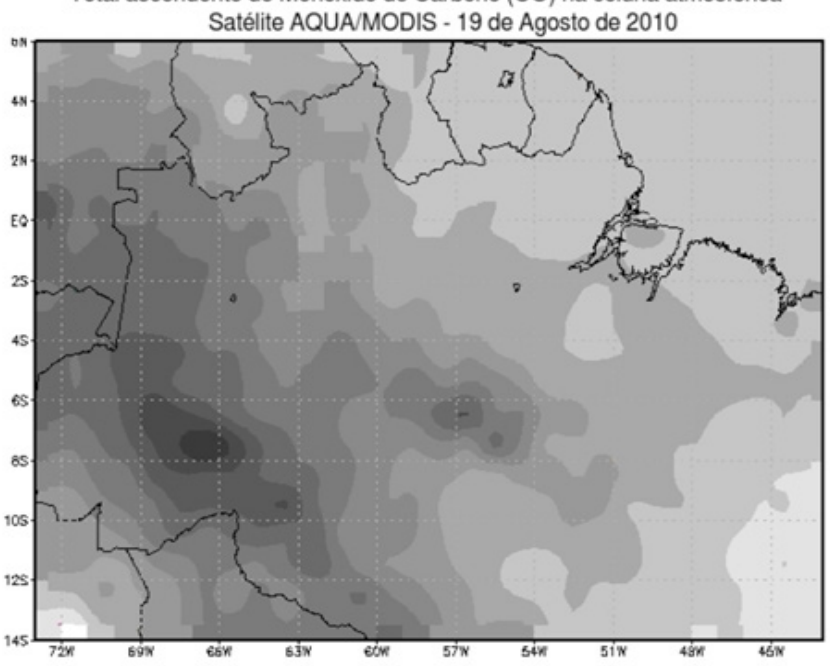

Figura 6 - Dispersão Total do monóxido de carbono sobre a região Amazônica gerada pelo modelo WRF/ CHEM e através dos dados do satélite AQUA
A sudeste da região, norte do estado do Mato Grosso e sul do Pará, é possível notar que a velocidade do vento a 10 metros da superfície obteve valores maiores se comparado a parte sudoeste, no limite entre o estados do Acre, sul do Amazonas e Rondônia. Isso fez com que a matéria gasosa emitida na queimada tivesse maior dispersão na região onde havia maior velocidade do vento. Isso pode ser comprovado nas imagens de sensoriamento remoto, onde vemos maiores concentrações sobre a região onde foi visto menores valores para velocidade do vento.

É importante ressaltar que o modelo não utilizou condição de background, isto é, antes do início da rodada não havia Monóxido de Carbono presente na atmosfera da região.

\subsection{Análise das concentrações máximas de mo- nóxido de carbono (CO)}

Uma análise sobre o CO emitido em uma queimada se faz necessária para a validação do trabalho, isto é, sabendo os fatores que influenciam na dispersão do poluente emitido, é avaliado a precisão que a mesma está sendo emitida, condiz com a realidade. Para isso foi avaliado algumas bibliografias com que registraram concentrações de $\mathrm{CO}$ em queimadas na região Amazônica.

Em Cordova, 2003 apud Artaxo et al., 2002 é mostrado concentrações de monóxido de carbono (CO) em Rondônia durante a estação chuvosa variam de 100 a 150 ppbv, enquanto que na estação de queimadas variam de 1.000 a 8.000 ppbv.

Em análise feita através do sistema de monitoramento operacional implementado no CPTEC/INPE usando o modelo de transporte 3D on-line CATT-BRAMS (Coupled Aerosol andTracer Transport model to the Brazilian developments on the Regional Atmospheric Modelling System), disponível em < meioambiente.cptec.inpe.br>. Foi observado para o dia 16 de Agosto de 2010 o nível de monóxido de carbono em todo o estado do Acre variando de 400 a 1250 ppbv em áreas afastadas aos pontos de emissão, com valores de concentração junto aos focos próximo a 2000 ppbv.

No experimento descrito por Sachse et al (1988) durante a Amazon Boundary Layer Experiment (ABLE 2A), foram feitas medições de $\mathrm{CO}$ utilizando um sensor laser sobre a bacia Amazônica. Foram medidas concentrações de 800 ppbv próximas a algumas camadas de plumas que foram identificadas.

\section{Conclusão}

$\mathrm{Na}$ análise da dispersão do poluente $\mathrm{CO}$ sob influência da direção e velocidade do vento a 10 metros de altura, o acoplamento da química com a meteorologia no modelo WRF/CHEM se mostrou eficiente em estimar as variações na concentração de CO.

É possível notar as mudanças diárias no padrão do 
vento tanto na velocidade quanto na direção, isso influi diretamente no transporte do gás. Onde no geral teve uma dispersão para o quadrante Oeste, com momentos de total calmaria ocasionando uma estagnação dos poluentes e altas concentrações próximo a região da queimada, chegando atingir valores superiores a 2000 ppbv nessas condições.

Foi possível verificar e confirmar o sentido da dispersão do Monóxido de carbono emitido na Região Amazônica no dia 19 de Agosto de 2011 através dos dados do satélite AQUA. Com isso, avaliou-se o desempenho do modelo WRF/CHEM no transporte do gás. É visto que o sentido sudeste-noroeste caracterizado pelo modelo é observado também nos dados de sensoriamento remoto, e ainda que a maior velocidade do vento em 10 metros a sudeste (nordeste do estado do Mato Grosso, e sul do Pará) da região da Amazônia contribuiu para uma maior dispersão do poluente em comparação a região entre o sul do estado do Amazonas, Acre e Rondônia. É possível observar em ambas imagens a dispersão do poluente para fora do território do brasileiro, chegando a outros países, como Colômbia e Peru.

Para a continuidade do estudo os autores pretendem fazer análises dos materiais (aerossóis) emitidos nas queimadas na Amazônia Legal, verificando os impactos na precipitação da região.

\section{Agradecimentos}

O primeiro autor agradece a CAPES pela concessão da bolsa em nível de mestrado e para o PROCAD (Programa Nacional de Cooperação Acadêmica) no DSA-INPE (Divisão de Satélites e Sistemas Ambientais - Instituto Nacional de Pesquisas Ambientais). Agradece também aos cientistas da missão MODIS e pessoal associado a NASA a disponibilização e produção de dados utilizados nesta pesquisa.

\section{Referências}

Acker, J. G., Leptoukh, G. Online Analysis Enhances Use of NASA Earth Science Data, Eos, Trans. AGU, Vol. $88, \mathrm{~N}^{\circ}$ 2, 9 January 2007, 14, 17 p.

Ambiente Acreano - Fumaça Já Afeta a Qualidade do Ar em Todo Acre. Disponível em: < http://ambienteacreano. blogspot.com.br/2010/08/fumaca-de-queimadas-ja-afetaqualidade.html> Acesso em 23 de Maio de 2013.

Andreae, M.: Biomass burning its history, use and distribution and its impact on environmental quality and global climate. In: J. S. Levine (ed.), Global Biomass Burning atmospheric, Climatic and Biospheric Implications, pp. 3-21, MIT Press, Cambridge, Mass, 1991.
Andreae, M., and Merlet, P. Emission of trace gases and aerosols from biomass burning, Global Biogeochemical Cycles, 15, 4, 955-966, 2001.

Aragão, L.E.O.C., Shimabukuro, Y. E., Lima, A., Anderson, L. O., Barier, N., Saatchi. Utilização de produtos derivados de sensores orbitais para o estudo de queimadas na Amazônia. Anais XIV Simpósio Brasileiro de Sensoriamento Remoto, Natal, Brasil, 25-30 abril 2009, INPE, p. 919-925.

Artaxo, P., Gatti L.V., Leal A. M. C., Longo K. M., Freitas, S. R., Lara L. L., Paulisquevis T. M., Procópio A. S., Rizzo L. V. Química atmosférica na Amazônia: A floresta e as emissões de queimadas controlando a composição da atmosfera amazônica. Vol. 35(2) 2005: 185-196 p.

Artaxo, P.; Química atmosférica na Amazônia: A floresta e as emissões de queimadas controlando a composição da atmosfera amazônica. Andreae, M. O.; Guenther, A.; Rosenfeld, D.; 2001. LBA Atmospheric Chemistry: Unveiling the lively interactions between the biosphere and the Amazonian atmosphere. In: IGBP Global Change Newsletter, LBA Special Issue, 12-15.

Artaxo, P.; Martins, J.V.; Yamasoe, M. A.; Procópio, A. S.; Pauliquevis, T. M.; Andreae, M. O.; Guyon, P.; Gatti, L. V.; Cordova Leal, A.M. 2002.Physical and chemical properties of aerosols in the wet and dry season in Rondônia,Amazonia. J. Geophys. Res., 107 (D20): 49.149.14 .

Cançado, J.E.D. A poluição atmosférica e sua relação com a saúde humana na região canavieira de Piracicaba - SP. Tese (Doutorado) - Faculdade de Medicina, Universidade de São Paulo. São Paulo, 2003, 201p.

Cordova, A. M.; 2003. Gases traço na Amazônia: Variabilidade sazonal e temporal de O3, NOx e CO em ambientes de pastagem e floresta. Tese de doutorado, Instituto Astronômico, Geofísico e de Ciências Atmosféricas da USP (IAG/USP), São Paulo, São Paulo, 268 pp.

Freitas, S. R. Modelagem Numérica do Transporte e das Emissões de Gases Traços e Aerossóis de Queimadas no Cerrado e Floresta Tropical da América do Sul. Tese de Doutoramento em Física Aplicada - Instituto de Física, Universidade de São Paulo, agosto de 1999. 204 p.

Freitas, S., K. Longo, M. Silva Dias, P. Silva Dias, R. Chatfield, E. Prins, P. Artaxo, G. Grell and F. Recuero. Monitoring the transport of biomass burning emissions in South America. Environmental Fluid Mechanics, Kluwer Academic Publishers, 2004 (em impressão, disponível eletronicamente em www.kluweronline.com/issn/15677419, na seção Articles in Advance). 
Freitas, S. R., Longo, K. M., Silva Dias, M., Silva Dias, P., Chatfield, R., Prins, E., Artaxo, P., Grell, G., and Recuero, F.: Monitoring the transport of biomass burning emissions in South America, Environmental Fluid Mechanics, doi:10.1007/s10652-005-0243-7, 5 (1-2), 135-167, 2005.

Freitas, S. R., Longo, K. M., and Andreae, M. O.: Impact of including the plume rise of vegetation fires in numerical simulations of associated atmospheric pollutants, Geophys. Res. Lett., 33, L17808, doi:10.1029/2006GL026608, 2006.

Freitas, S. R., Longo, K. M., Chatfield, R., Latham, D., Silva Dias, M. A. F., Andreae, M. O., Prins, E., Santos, J. C., Gielow, R., and Carvalho Jr., J. A.: Including the subgrid scale plume rise of vegetation fires in low resolution atmospheric transport models, Atmos. Chem. Phys., 7, 3385-3398, doi:10.5194/acp-7-3385-2007, 2007.

Gash, J. H. C.; Nobre, C. A.; Roberts, J. M.; Victoria, R. L. 1996. Amazonian Deforestation and climate. John Wiley and Sons, Inglaterra.

Giovanni - Interactive Visualization and Analysis. Disponível em: <http://disc.sci.gsfc.nasa.gov/giovanni $>$ Acesso constante.

Grell, G., Freitas, S. R., Stuefer, M. , Fast J.: Inclusion of biomass burning in WRF-Chem: impact of wildfires on weather forecasts, Atmos. Chem. Phys. Discuss., 10, 30613-30650, 2010

IBGE - Instituto Brasileiro de Geografia e Estatística. 2010. Disponível em: < http://www.ibge.gov.br/estadosat/ perfil.php?sigla=am > Acesso em: 14 Jun. 2012.

INPE - Instituto Nacional de Pesquisas Espaciais, 2012. Portal de Monitoramento de Queimadas e Incêndios. Disponível em http://www.inpe.br/queimadas. Acesso em: Abril de 2013

INPE - Instituto Nacional de Pesquisas Espaciais, 2012. Portal de Qualidade do Ar. Disponível em http:// meioambiente.cptec.inpe.br/. Acesso em: Maio de 2013.

Longo, K. M.; Freitas, S. R.; Setzer, A.; Prins, E.; Artaxo, P.; Andreae, M. O. The Coupled Aerosol and Tracer Transport model to the Brazilian developments on the Regional Atmospheric Modeling System (CATTBRAMS). Part 2: Model sensitivity to the biomass burning inventories. In press: ACPD/EGU MS\# 0167, 2007.

EMBRAPA - Queimadas na Amazônia brasileira. In: Comunicado Técnico 18. ISSN 1415-2118. Campinas, SP. Abril, 2006.
Sachse, G. W.; Harriss, R. C.; Fishman, J.; Hill, G. F. and Cahoon, D. R. Carbon monoxide over the Amazon Basin during the 1985 dry season. J. Geophys. Res. 93, 1422-1430. 1988. 